rather harder than lipomata but elastic, freely movable, painless, and have appeared stationary for over twelve months. She is not losing weight or showing any other signs of dissemination of melanomata.

The diagram of the family tree shows the sequence through the generations, but apart from that striking feature there are other points of interest, viz., the ages; the average age for the five recorded cases being 28.6 years, and the fact that all have died from secondary deposits except one, who after ten years has now shown evidence of dissemination.

It is fortunate that the cases have all come to Moorfields and that, therefore, the clinical and pathological records can be assembled. As Moorfields Research Scholar I have been able to collect the records and in addition to see the earlier reports on the family and Sir John Parsons's note on the history. I am indebted to Sir Arnold Lawson and Mr. Hudson for permission to add the two later cases.

\title{
A FAMILY WITH BLUE SCLEROTICS
}

BY

Major J. N. Duggan, D.O.(Oxon), F.C.P.S., L.M. \& S.(Bom.), AND

B. P. Nanavati, D.O.M.S., M.R.C.S.(Eng.)

STray cases of blue sclerotics are often met with and occasionally found running in a family, but a case of blue sclerotic, described below, with a light blue discolouration of half of the face on the same side as the affected eye is of less frequent occurrence. We therefore include a description of this case along with the hereditary group.

The following are the notes on the various cases examined:

A Mahomedan boy, aged 11 years, was brought to the Sir C. J. Eye Hospital, Bombay, to have his vision tested, as he had poor sight. On noticing the blue colour of the sclerotics his father, who had accompanied him and whose eyes were normal, was questioned about their presence in the family. On his answering in the affirmative the affected persons were examined.

In the family these could be traced only on the side of the boy's mother. He had two sisters who were also affected. The mother of the three children had also blue sclerotics. Her brother who was slightly affected and both her sisters had the same colour about their eyes. The brother had three sons with normal eyes, but both the sisters had the abnormality in their families which 
consisted of two boys and two girls in each case. On inquiring further it was found that the maternal grandmother of the children, who seemed to be the starting point, was also similarly affected. This brought out the interesting points that the abnormality was transmitted through the affected females to the descendants of the family and was dying out through the only male member who was slightly affected. There was no consanguinity of parents. The family tree is as follows :

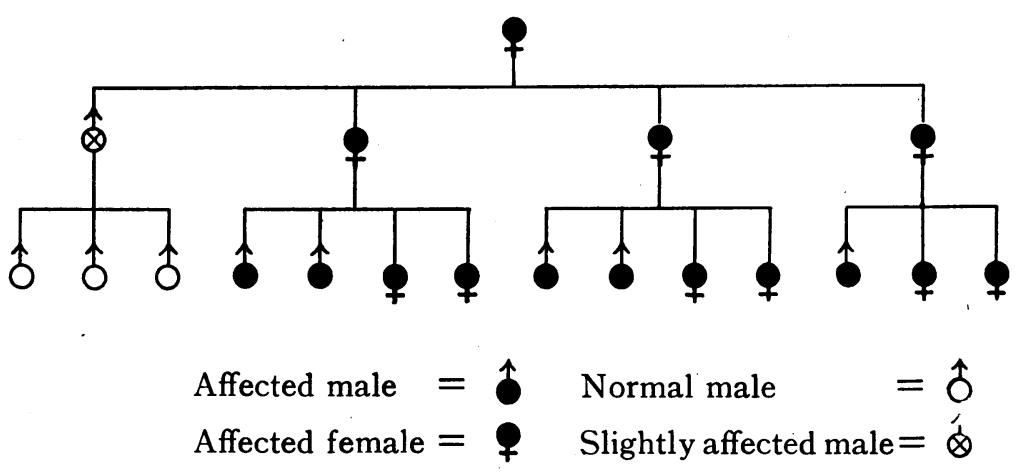

The details of the clinical examination of some of the cases are as follow :

(1) A boy, aged 11 years, had both his sclerotics of deep blue colour. The greatest depth of colour was noticed in a circular zone one $\mathrm{cm}$. wide round the limbus. The anterior perforating ciliary vessels were well marked out in the right eye with brownish pigmentation at the sites of perforation. He had myopia of moderate degree and visual acuity was $6 / 6$ with sphero-cylindrical glasses. Iris was of deep brown colour. Fundi were normal.

(2) The colour of both the sclerotics of a girl, aged 10 years, was deep blue. The sclero-corneal junction stood out prominently as a white circle. Sites of the perforating ciliary vessels were marked out by deep brown pigmentation. On the surface of the right eye there were three small irregularly scattered patches of brown pigment. Vision and fundi were normal. Colour of iris was brown.

(3) This patient was a girl, aged 9 years. The colour of both the sclerotics was pale blue with scattered patches of deeper tint. Vision and fundi were normal. Colour of iris was brown.

(4) The mother of the above-mentioned children had a zone of deep blue sclerotics varying irregularly from $0.5 \mathrm{~cm}$. to $1 \mathrm{~cm}$. in width round the limbus and gradually fading into the light blue colour of the remaining sclera. Vision and fundi were normal. Iris was of dark brown colour. 
The case of blue sclerotic with blue discolouration of the face mentioned in the beginning of the article was in no way connected with the hereditary group already described. It was noted in a Multani girl of a blonde type, aged 9 years, the sclerotic of whose right eye was of deep blue colour and who had an adherent leucoma of the same eye. The right half of the face had a faint blue discolouration. The discoloured area extended a little beyond the middle line, nearly up to it on the lower jaw and as far as the right ear. The right side of the nose and upper lid had escaped the discolouration. At the periphery the blue colour gradually faded away into the normal tint of the skin. The skin in the affected area was not thickened and did not show any dilated blood-vessels. The eye except for the adherent leucoma and blue sclerotic was of normal size and shape. No other member of the family was similarly affected though she herself was born with it. Before a photograph of the face could be arranged for she disappeared.

\section{Remarks}

(1) All cases of the hereditary type were bilateral.

(2) No evidence of fragilitas ossium, deafness, or congenital syphilis could be obtained.

(3) All cases were free from error of refraction except one that was myopic.

(4) All the affected members of the family were females with only one exception, that being a slightly affected male.

(5) The affection was transmitted through females to the descendants and was dying out through the only male member of the family.

\section{A FAMILY WITH ANIRIDIA}

$\mathrm{BY}$

Major J. N. DugGan, D.(O.(Oxon), F.C.P.S., L.M. \& S.(Bom.), AND

B. P. Nanavati, D.O.M.S., M.R.C.S.(Eng.)

IT is not often that one comes across cases of aniridia, much less so as a familial disease, and hence the following cases are considered worthy of record :

Family History.-All the affected children were the offspring of the third wife of their father, those by the first two being quite 